\title{
Hemodynamic Changes and Clinical Outcomes after the Intra/Extracardiac Fenestrated Fontan Procedure
}

\author{
Naveen Menon, Chihyang Lin, Cesar I. Mesia, Achintya Moulick, Randy Stevens, \\ Vicki Mahan* \\ Drexel University College of Medicine and Department of Pediatric Cardiothoracic Surgery, St. Christopher's \\ Hospital for Children, Philadelphia, PA, USA \\ Email: *mahan_vicki@yahoo.com
}

How to cite this paper: Menon, N., Lin, C., Mesia, C.I., Moulick, A., Stevens, R. and Mahan, V. (2018) Hemodynamic Changes and Clinical Outcomes after the Intra/ Extracardiac Fenestrated Fontan Procedure. World Journal of Cardiovascular Surgery, 8, 29-39.

https://doi.org/10.4236/wjcs.2018.82004

Received: January 12, 2018

Accepted: February 8, 2018

Published: February 12, 2018

Copyright $\odot 2018$ by authors and Scientific Research Publishing Inc. This work is licensed under the Creative Commons Attribution International License (CC BY 4.0).

http://creativecommons.org/licenses/by/4.0/

(c) (i) Open Access

\begin{abstract}
Background: The primary concern of the Fontan procedure has been atrial arrhythmias, but little attention has been given to hemodynamic changes. This study's purpose was to examine the hemodynamics and arrhythmias of the Intra/extracardiac fenestrated Fontan, and determine any advantages/disadvantages of this newer Fontan procedure. Methods: Patients undergoing the intra/ extracardiac fenestrated Fontan procedure at this institute between December 2009 and June 2017 were included in this retrospective evaluation of preoperative and postoperative hemodynamics during cardiac catheterization. End Diastolic Pressure (EDP), Pulmonary Artery Pressure (PAP), Left Atrial Pressure (LAP), and Transpulmonary Gradient (TPG) were the hemodynamic markers of interest. Occurrence of arrhythmias was also examined preoperatively, less than 2 weeks postoperatively, and greater than 2 weeks postoperatively. Morbidities and mortalities were also evaluated. Results: Fourteen patients underwent the procedure between December 2009 and June 2017. Preoperative data was available in all of these patients. Postoperative data was partially incomplete for atrial arrhythmias and hemodynamic data. EDP rose from $9.29 \pm$ $3.50 \mathrm{mmHg}$ to $10.33 \pm 3.12 \mathrm{mmHg}$. PAP rose from $12.57 \pm 2.50 \mathrm{mmHg}$ to $14.27 \mathrm{mmHg}$. TPG dropped from $5.00 \pm 2.29 \mathrm{mmHg}$ to $3.25 \pm 1.67 \mathrm{mmHg}$. LAP rose from $7.57 \pm 2.87 \mathrm{mmHg}$ to $10.30 \pm 1.95 \mathrm{mmHg}$. Atrioventricular valve (AVV) regurgitation remained the same pre and postoperatively. 8 of 13 patients developed arrhythmias at less than 2 weeks postoperatively, and 5 of 12 patients developed arrhythmias at greater than 2 weeks postoperatively. There were no morbidities or mortalities. Conclusions: We found a statistically significant change in EDP, PAP, LAP, and TPG levels. It is unclear whether this is an advantage or disadvantage. The significance of these changes is unclear
\end{abstract}


in this small population of patients. Further evaluation of hemodynamics, arrhythmias, morbidities and mortalities associated with the intra/extracardiac Fontan procedure is needed.

\section{Keywords}

Fontan, Functionally Univentricular Heart, Circulatory Hemodynamics, Hypoplastic Left Heart Syndrome, Congenital Heart Disease (CHD)

\section{Introduction}

The palliative procedure for patients with a single functioning ventricle has undergone several evolutionary changes throughout the years. The Fontan technique developed from the Atriopulmonary Fontan [1] [2], which worked effectively, but exposed a large portion of atria to elevated venous pressure, leading to a severely dilated, hypertrophied right atrium, and a higher risk of late supraventricular arrhythmias [3]. The resultant elevated pulmonary venous pressure and obstruction of the pulmonary-venous pathway were also concerning [4].

These shortcomings led to the development of the Lateral Tunnel technique [5], which functioned by placing a baffle inside the right atrium, directing inferior vena cava blood into the pulmonary artery. The procedure avoids pulmonary venous obstruction (even at high pressures of the systemic venous baffle) and also exposes a smaller area of right atrial wall to the high pressure, thus reducing the risk of supraventricular tachycardia [1] [5]. During this time, a technique of baffle fenestration was developed, allowing for right to left shunting, maintaining cardiac output and limiting right atrial pressure [6].

The third generational technique was the Extracardiac Fontan, which eliminated exposure of the atrial wall to the Fontan circuit's elevated venous pressure. This procedure struggled to maintain fenestration patency, and the true efficacy of fenestration began to be questioned [7]. However, a decrease in fenestration usage has ultimately lead to an increased duration of pleural effusions (10 days \pm 12 days with fenestration, 13.2 days \pm 8.8 days without fenestration) [8]. In addition, while the purpose of the Extracardiac conduit was to lower the incidence of arrhythmias, many studies have shown the incidence of post-operative arrhythmias to be comparable to the Lateral Tunnel [9] [10]. Some studies have also shown an association between the use of the Extracardiac conduit and a higher incidence of sinus node dysfunction [11].

Each modification from the original Fontan/Kreutzer procedure has yielded significant advantages and disadvantages. In comparison to the Lateral Tunnel procedure, the Extracardiac Fontan is a simpler procedure that minimizes suture lines, and can be performed without cardiopulmonary bypass [12] and cross clamping, and does not yield high-pressure atrial tissues. However, this procedure's disadvantages are that fenestration is difficult, growth potential is limited, and catheter access to the atrium cannot be achieved. The Lateral Tunnel improved upon 
this procedure by allowing for easier fenestration and catheter access to the atrium, but also still had setbacks of a heavier atrial suture burden, a more readily inducible Supraventricular Tachycardia (SVT), and a thin strip of highly pressurized atrial tissue.

The newest modification of this procedure, the Intra/extracardiac conduit with fenestration, is expected to combine the benefits of the previous Fontan techniques, and improve upon these clinical outcomes. The procedure avoids injury to the sinus node artery and crista terminalis, and allows for easier fenestration and minimal disruption of atrial conduction pathways [1]. Due to this technique's novelty, prior data on the topic is scarce. We provide insight on this topic by examining clinical outcomes of patients who have undergone the Intra/extracardiac fenestrated Fontan during their first year of follow up.

\section{Patients and Methods}

\subsection{Description of Procedure}

The intra/extracardiac fenestrated Fontan procedure is done with the patient on cardiopulmonary bypass with the heart arrested. A transverse incision is made in the lower right atrium and a ringed conduit sewn to the inferior vena cava at its opening into the right atrium. A $4 \mathrm{~mm}$ punch is then used to create the fenestration in the conduit. The right atriotomy that is present is sutured to the ringed conduit leaving the fenestration within the right atrium. An incision is then made in the anterior wall of the superior vena cava-pulmonary artery connection and the proximal ringed conduit is anastomosed to this opening.

\subsection{Study Sample}

The institutional review board (IRB) of Drexel University College of Medicine approved the study. Informed consent was waived, as there was minimal risk nature to the patient.

All patients who underwent the intra/extracardiac fenestrated Fontan procedure at St. Christopher's Hospital for Children between December 2009 and June 2015 were included. The median (IQR) age at Fontan was 31 months $(27.25,37.75$ months). Arrhythmia and hemodynamic data was extracted from the pediatric cardiac surgery database. Preoperative hemodynamic data includes all 14 patients, and postoperative hemodynamic data was available for 9 patients for End Diastolic Pressure (EDP), 11 patients for Pulmonary Artery Pressure (PAP), 8 patients for Transpulmonary Gradient (TPG), 10 patients for Left Atrial Pressure (LAP), and 9 patients for Atrioventricular Valve (AVV) regurgitation. Presence or absence of preoperative and/or postoperative arrhythmias was available for all patients preoperatively and postoperatively.

\subsection{Assessment of Outcomes}

Rhythm data was obtained by analysis of available electrocardiograms and interpretation was by the pediatric cardiology electrophysiologist. Pre and post- 
operative data from these cases were compiled and statistically analyzed. The presence of abnormal arrhythmias was determined before operation, at less than 2 weeks after operation, and greater than 2 weeks after operation. A persistence or emergence of atrial arrhythmias postoperatively would represent a drawback of this procedure, while a reduction, or a lesser incidence in comparison to previous literature, would suggest an advantage.

Cardiac catheterization was completed in all patients before operation. Postoperative hemodynamic data was collected at least 1 year after the procedure. 95\% confidence intervals for differences in pre and postoperative values were calculated for hemodynamic values. Hemodynamic endpoints were the primary endpoints and included End Diastolic Pressure (EDP), Pulmonary Artery Pressure (PAP), Left Atrial Pressure (LAP), and Transpulmonary Gradient (TPG). Arrhythmias and other morbidities were secondary endpoints. Mean ventilation time, chest tube drainage, and length of hospital stay were also included in the descriptive characteristics. Changes in these parameters may suggest physiologic end-points that would result in fewer complications resulting from the procedure.

\subsection{Statistical Analysis}

Pre and postoperative data for various hemodynamic values were compared for all patients who had postoperative catheterization data available, and confidence intervals for the differences in mean values were generated. Due to the small sample size of the study, linear regression analysis for predictive variables and other statistical procedures were avoided. All descriptive information and hemodynamics are reported in tables below.

\section{Results}

\subsection{Descriptive Information}

Inclusion criteria consisted of patients who underwent the Intra/extracardiac fenestrated Fontan between December 2009 and June 2015. Fourteen patients were included in the study. No mortalities were observed. Descriptive information is shown in Table 1.10 of 14 patients were male. Diagnoses included Doublet Outlet Right Ventricle, Tricuspid Atresia, Pulmonary Atresia, Pulmonary Stenosis, Ventricular Septal Defect, Hypoplastic Left Heart Syndrome, Transposition of the Great Arteries, Complete Atrioventricular Canal Defect, Double Inlet Left Ventricle, and Heterotaxy. Median (IQR) age at Fontan (months) was 31 (27.25, 37.75). Median (IQR) weight at Fontan $(\mathrm{kg})$ was $12.4(11.2,13.6)$, and BSA $\left(\mathrm{m}^{2}\right)$ was $0.55(0.51,0.60)$. Median (IQR) cardiopulmonary bypass time (min) was 102.5 (96.75, 110), and cross clamp time $(\min )$ was $53.5(50,61.5)$. Median (IQR) Intensive Care Unit stay (days) was $12(9,21)$, length of hospital stay (days) was $14.5(10.3,19.8)$, and median length was chest tube drainage (days) of $6.5(3,8)$. Median (IQR) time from catheterization to follow up (months) was 41 $(18,47)$ 
Table 1. Preoperative, intraoperative, and postoperative descriptive characteristics. Data was taken from all 14 patients unless otherwise indicated.

\begin{tabular}{|c|c|}
\hline Characteristic & Patients $(n=14)$ \\
\hline Male, n (\%) & $10(71 \%)$ \\
\hline Females, n (\%) & $4(28.5 \%)$ \\
\hline \multicolumn{2}{|l|}{ Diagnoses, $\mathrm{n}(\%)$} \\
\hline Double Outlet Right Ventricle & $6(42 \%)$ \\
\hline Tricuspid Atresia & $5(35 \%)$ \\
\hline Pulmonary Atresia & $3(21 \%)$ \\
\hline Pulmonary Stenosis & $1(7 \%)$ \\
\hline Ventricular Septal Defect & $1(7 \%)$ \\
\hline Hypoplastic Left Heart Syndrome & $1(7 \%)$ \\
\hline Transposition of the Great Arteries & $1(7 \%)$ \\
\hline Complete Atrioventricular Canal Defect & $1(7 \%)$ \\
\hline Double Inlet Left Ventricle & $1(7 \%)$ \\
\hline Heterotaxy & $3(21 \%)$ \\
\hline 2 or more of the above diagnoses & $7(50 \%)$ \\
\hline \multicolumn{2}{|l|}{ Preoperative } \\
\hline Age at Fontan (months), Median (IQR) & $31(27.5-37.5)$ \\
\hline Weight at Fontan $(\mathrm{kg})$, avg \pm std. dev & $12.6 \pm 2.1$ \\
\hline BSA at Fontan $\left(\mathrm{m}^{2}\right)$ & $0.56 \pm 0.1$ \\
\hline Abnormal Arrhythmias & $1(8 \%)$ \\
\hline \multicolumn{2}{|l|}{ Intraoperative } \\
\hline CPB Time (minutes), Median (IQR) & $103.1 \pm 12.5$ \\
\hline Cross Clamp Time (minutes) & $55.1 \pm 9.8$ \\
\hline Fenestration & $100 \%$ \\
\hline \multicolumn{2}{|l|}{ Postoperative } \\
\hline Ventilation Time (days), Median (IQR) & $0(0,1)$ \\
\hline ICU Stay (days), Median (IQR) & $12(9,21)$ \\
\hline Length of Stay (days), Mean (IQR) & $14.5(10.3,19.8)$ \\
\hline Length of Follow-Up to Date (months), Median (IQR) & $41(19,47)$ \\
\hline Chest Tube Drainage (days), Median (IQR) & $6.5(3,8)$ \\
\hline \multicolumn{2}{|l|}{ Abnormal Arrhythmias } \\
\hline Pre-fontan & $1 / 13(8 \%)$ \\
\hline$<2$ Weeks after Fontan & $8 / 13(61 \%)$ \\
\hline$>2$ Weeks after Fontan & $5 / 12(41 \%)$ \\
\hline \multicolumn{2}{|l|}{ Inotrope Score, Median (IQR) } \\
\hline 24 Hours & $5(5,7.5)$ \\
\hline 48 Hours & $0(0,3)$ \\
\hline \multicolumn{2}{|l|}{ Vasoactive Inotropic Score (IQR) } \\
\hline 24 Hours & $13(10,15)$ \\
\hline 48 Hours & $10(5,10)$ \\
\hline Mortalities & $0(0 \%)$ \\
\hline
\end{tabular}




\subsection{Arrhythmias}

Patient arrhythmia data was available for patients preoperatively, for 13 patients less than 2 weeks post operatively, and for 12 patients for more than 2 weeks postoperatively. Only one patient had abnormal arrhythmias prior to the operation. 8 out of 13 patients (61\%) developed arrhythmias after the operation, but for only 5 out of 12 patients did this persist for greater than 2 weeks.

\subsection{Hemodynamics}

Hemodynamic information is presented in Table 2 and Table 3. All patients had preoperative data. Postoperative data included 9 patients for end diastolic pressure (EDP), 11 patients for pulmonary artery pressure (PAP), 8 patients for transpulmonary gradient (TPG), 10 patients for left atrial pressure (LAP) and 9 patients for atrioventricular valve (AVV) regurgitation. EDP rose from 9.29 \pm 3.50 $\mathrm{mmHg}$ to $10.33 \pm 3.12 \mathrm{mmHg}$. PAP rose from $12.57 \pm 2.50 \mathrm{mmHg}$ to 14.27 mmHg. TPG dropped from $5.00 \pm 2.29 \mathrm{mmHg}$ to $3.25 \pm 1.67 \mathrm{mmHg}$. LAP rose from $7.57 \pm 2.87 \mathrm{mmHg}$ to $10.30 \pm 1.95 \mathrm{mmHg}$. AVV regurgitation remained the same pre and postoperatively. All hemodynamic values were significant, based on a $95 \%$ confidence interval for the difference of means for pre and postoperative values.

\section{Discussion}

\subsection{Arrhythmias}

Rhythm abnormalities remain a primary concern for patients with a single functioning ventricle. While long-term survival from the Fontan procedure is improving, atrial flutter and other arrhythmias are known to frequently develop postoperatively and are often fatal [13] [14] [15]. Atrial arrhythmias have been attributed to injury of the crista terminalis and Eustachian ridge [16] [17]. Avoidance of an incision injury to the sinus node, sinus node artery, and crista terminalis [1] is a goal of the Intra/extracardiac fenestrated Fontan procedure and should result in a lower incidence of atrial arrhythmias relative to the Lateral Tunnel and the Extracardiac procedures. Sinha and colleagues have shown a lower incidence of atrial arrhythmias in their population of patients undergoing this procedure [18]. In particular, the intra/extracardiac fenestrated Fontan technique was shown to have a $27 \%$ incidence of abnormal arrhythmia at greater than two

Table 2. Fontan hemodynamic information.

\begin{tabular}{lcc}
\hline & Before Procedure & After Procedure \\
\hline EDP (mmHg), mean \pm std. deviation & $9.29 \pm 3.50$ & $10.33 \pm 3.12$ \\
PAP (mmHg), mean \pm std. deviation & $12.57 \pm 2.50$ & $14.27 \pm 2.23$ \\
TPG (mmHg), mean \pm std. deviation & $5.00 \pm 2.29$ & $3.25 \pm 1.67$ \\
LAP (mmHg), mean \pm std. deviation & $7.57 \pm 2.87$ & $10.3 \pm 1.95$ \\
AVV Regurgitation, median (range) & $1(0-2)$ & $1(0-2)$
\end{tabular}


Table 3. 95\% confidence intervals for the difference of hemodynamic means before and after Fontan.

\begin{tabular}{cc}
\hline & CI \\
\hline EDP (mmHg) & $1.04 \pm 0.837^{\star}$ \\
PAP (mmHg) & $1.70 \pm 0.540^{\star}$ \\
TPG (mmHg) & $-1.75 \pm 0.520^{\star}$ \\
LAP (mmHg) & $2.73 \pm 0.555^{\star}$ \\
\hline
\end{tabular}

* = Statistically significant.

weeks postoperatively, a lower percentage than that of the Lateral Tunnel (58\%). Worsening of rhythm comparing rhythm preoperatively with more than 2 weeks postoperatively yielded a statistically significant result favoring the Intra/extracardiac technique over the Lateral Tunnel technique ( $\mathrm{p}=0.015)$. In addition, while not statistically significant, the Intra/extracardiac technique also trended to having lower incidences than the Extracardiac technique at 2 weeks postoperatively (36\%). However, we found an increased incidence of atrial arrhythmias in our study group, showing that this issue may still be problematic. It is possible that this advantage of the intra/extracardiac is not as profound as once thought. However, it should be noted that the incidence of atrial arrhythmias was decreased at greater than 2 weeks postoperatively, than at less than 2 weeks postoperatively, showing that they could be self resolving with time. Our patients did trend to having a lower Vasoactive Inotropic Score (VIS) from 24 hours to 48 hours, which is beneficial since a high maximum VIS has been shown to be a strong predictor of clinical outcome (death, cardiac arrest, mechanical circulatory support, etc.) in this patient population [19].

Arrhythmias are important to monitor not only for the immediate danger with which they present, but also for that of the long term. Carins et al. studied the long term outcomes of various Fontan patients in Australia and New Zealand, and found that the development of any first onset arrhythmia, bradyarrhythmia or tachyarrhythmia to be independently associated with Fontan failure (defined as death, heart transplantation, Fontan takedown or conversion, protein-losing enteropathy, plastic bronchitis, or New York Heart Association functional class III or IV at follow-up) [20]. While the literature shows that the intra/extracardiac fenestrated Fontan yields lower incidences of arrhythmias in comparison to prior Fontan procedures, the incidence found postoperatively still remains at a level that is not ideal, and various complications are expected. Thus, a careful watch should still be given to those patients who develop arrhythmias.

Longer-term evaluation is needed to further refine our understanding and the role of this new technique in those patients with single ventricle physiology. In order to adequately compare the incidences of postoperative arrhythmias, the Intra/extracardiac technique should be compared to the Lateral Tunnel and Extracardiac techniques on a much larger scale, as seen in the Sinha study. In addition, our study has a much higher prevalence of male patients, possibly masking 
a role of sex in the postoperative outcome of the Fontan patient.

\subsection{Hemodynamics and Morbidities}

Postoperative end diastolic pressure (EDP), mean pulmonary artery pressure (MPAP), mean left atrial pressure (MLAP), and transpulmonary gradient (TPG) were all statistically different from their preoperative values. EDP, MPAP, and MLAP increased after the operation while the TPG decreased. Although these hemodynamic values were statistically significant, the magnitude to which the pressures changed was not as impressive. Most of the hemodynamic values remained in their normal ranges, but trended toward their upper limits, while TPG trended toward its lower limit. The importance of these findings is unclear in this limited study and deserves further evaluation as changes in these postoperative parameters may help us to further refine therapies directed at the Fontan physiology.

Past studies on Fontan hemodynamics can give us insight into the clinical implications of these findings. The main complications after the Fontan procedure have been described as multi-end-organ congestion due to high venous pressure, low cardiac output leading to chronic heart failure, and mild but significant hypoxia [21]. In addition, elevated CVP and low arterial blood oxygen saturation have correlated with mortality in both early and late Fontan patients, while a greater end-diastolic volume index, along with a lower ejection fraction, has also been associated with increased mortality in early term patients [22]. With EDP and LAP pressures increasing, it is possible that this operation could lead to cardiac dysfunction, lowered cardiac output, and death, and so these values should be monitored on a long-term scale. In addition, a heightened pulmonary artery pressure could be a result of an increased pulmonary resistance, and could worsen the patient's hypoxia. The lowered TPG supports the notion of increased hypoxia in these patients. Thus, these hemodynamic changes represent a possible disadvantage in this procedure, but a comparison must be made to prior Fontan procedures to confirm this.

Other hemodynamic information is available in the world literature regarding the different Fontan procedures. Computational fluid dynamic studies conducted by Tain-Yen Hsia compared the directionality and energy losses between the intra atrial tube, lateral tunnel, extracardiac conduit with left pulmonary anastomoses, and extracardiac conduit with right pulmonary anastomoses procedures [23]. They concluded that the extracardiac conduit angled toward the left lung resulted in the lowest level of energy loss, and thus had the best fluid dynamic performance. While they also noted that computational modeling could not predict postoperative failure, it is important to observe the importance of local geometry in these Fontan procedures. A next step in this series would be to compare the geographic energy saving or expending capabilities of the Intra/extracardiac Fontan in comparison to the previous generations. Another study observed theoretical cardiac cycle-averaged power loss and hepatic flow distribution after modeling identical physiologic pulsatile caval flow and time-resolved pulmonary ar- 
tery flow. The study compared lateral tunnel, extracardiac conduit, intra/extracardiac conduit, and direct cavopulmonary connection (DCPC) Fontan pathway and determined which technique had the optimal tradeoff. It was revealed that, again, the extracardiac conduit yielded the lowest energy lost, but the poorest hepatic flow distribution. In the lateral tunnel and intra/extracardiac conduit, the results were reversed. The DCPC, however, yielded the best tradeoff in these two variables, and thus should also be included in future examinations and comparisons of these procedures.

\subsection{Limitations}

This study is limited by its retrospective nature and small sample size. In addition, there have not been studies directly comparing the pressures we have observed in this review among the different Fontan procedures, and so we cannot conclude the superiority of this procedure over the Lateral Tunnel and Extracardiac procedures. We therefore recommend pursuing this goal on a larger scale. We also suggest a more longitudinal study as it would be beneficial to observe the effects of these hemodynamic changes later in life. In addition to the hemodynamics observed in this study, we recommend also including those associated with higher mortality, such as CVP and $\mathrm{SaO}_{2}$ levels, as well as those aforementioned variables that other studies have examined.

\section{Conclusion}

Improved management of patients with Fontan physiology requires a better understanding of postoperative hemodynamics and arrhythmias. Even with the development of new Fontan techniques, cardiac complications are still arising. A more focused examination towards the hemodynamic endpoints seen in this study may help in identifying the physiological causes of these complications. Our patients exhibited, for the most part, normal postoperative hemodynamics, but EDP, LAP, and PAP values trended towards their upper normal limits, while TPG trended towards its lower limit. It is unclear whether these changes are an advantage or a disadvantage in this small population of patients. While we have shown statistically significant changes, further evaluation of postoperative hemodynamics after the Intra/extracardiac fenestrated Fontan procedure is needed in a larger patient population and on a longitudinal scale. In addition, postoperative arrhythmias continue to remain a problem in this patient population, and should be monitored longitudinally, as they are at high risk for complications.

\section{References}

[1] Jonas, R. (2011) The Intra/Extra Cardiac Conduit Fenestrated Fontan. Seminars in Thoracic and Cardiovascular Surgery. Pediatric Cardiac Surgery Annual, 14, 11-18. https://doi.org/10.1053/j.pcsu.2011.01.010

[2] Fontan, F. and Baudet, E. (1971) Surgical Repair of Tricuspid Atresia. Thorax, 26, 240-248. https://doi.org/10.1136/thx.26.3.240

[3] Mavroudis, C., Deal, B., Backer, C., et al. (2007) J. Maxwell Chamberlain Memorial 
Paper for Congenital Heart Surgery: 111 Fontan Conversions with Arrhythmia Surgery: Surgical Lessons and Outcomes. The Annals of Thoracic Surgery, 84, 1457-1465. https://doi.org/10.1016/j.athoracsur.2007.06.079

[4] Fontan, F., Kirklin, J.W., Fernandez, G., et al. (1990) Outcome after a "Perfect" Fontan Operation. Circulation, 81, 1520-1536. https://doi.org/10.1161/01.CIR.81.5.1520

[5] Jonas, R. and Castaneda, A. (1988) Modified Fontan Procedure: Atrial Baffle and Systemic Venous to Pulmonary Artery Anastomotic Techniques. Journal of Cardiac Surgery, 3, 91-96. https://doi.org/10.1111/j.1540-8191.1988.tb00228.x

[6] Bridges, N., Lock, J. and Castaneda, A. (1990) Baffle Fenestration with Subsequent Transcatheter Closure: Modification of the Fontan Operation for Patients at Increased Risk. Circulation, 82, 1681-1688. https://doi.org/10.1161/01.CIR.82.5.1681

[7] Thompson, L., Petrossian, E., McElhinney, D., et al. (1999) Is It Necessary to Routinely Fenestrate an Extracardiac Fontan? Journal of the American College of Cardiology, 34, 539-544. https://doi.org/10.1016/S0735-1097(99)00228-4

[8] Fu, S., Feng, Z. and Dietmar, S. (2010) Factors Influencing Pleural Effusion after Fontan Operation: An Analysis with 95 Patients. Chinese Medical Science Journal, 25, 38-43. https://doi.org/10.1016/S1001-9294(10)60018-1

[9] Fiore, A., Turrentine, M., Rodefeld, M., et al. (2007) Fontan Operation: A Comparison of Lateral Tunnel with Extracardiac Conduit. The Annals of Thoracic Surgery, 83, 622-629. https://doi.org/10.1016/j.athoracsur.2006.09.070

[10] Hakacova, N., Lakomy, M. and Kovacikova, L. (2008) Arrhythmias after Fontan Operation: Comparison of Lateral Tunnel and Extracardiac Conduit. Journal of Electrocardiology, 41, 173-177. https://doi.org/10.1016/j.jelectrocard.2007.10.007

[11] Kumar, S., Rubinstein, C., Simsic, J., et al. (2003) Lateral Tunnel versus Extracardiac Conduit Fontan Procedure: A Concurrent Comparison. The Annals of Thoracic Surgery, 76, 1389-1396. https://doi.org/10.1016/S0003-4975(03)01010-5

[12] McElhinney, D., Petrossian, E., Reddy, M. and Hanley, F. (1998) Extracardiac Conduit Fontan Procedure without Cardiopulmonary Bypass. The Annals of Thoracic Surgery, 66, 826-1828. https://doi.org/10.1016/S0003-4975(98)00928-X

[13] d'Udekem, Y., Iyengar, A., Galati, J., Forsdick, V., Weintraub, R., et al. (2014) Redefining Expectations of Long-Term Survival after the Fontan Procedure: Twenty-Five Years of Follow-Up from the Entire Population of Australia and New Zealand. Circulation, 130, 32-38. https://doi.org/10.1161/CIRCULATIONAHA.113.007764

[14] Blaufox, A., Sleeper, L., Bradley, D., Breitbart, R., Hordof, A. Kanter, R., et al. (2008) Functional Status, Heart Rate, and Rhythm Abnormalities in 521 Fontan Patients 6 to 18 Years of Age. The Journal of Thoracic and Cardiovascular Surgery, 136, 100-107. https://doi.org/10.1016/j.jtcvs.2007.12.024

[15] Fishberger, S.B., Wernovsky, G., Gentles, T.L., Gauvreau, K., Burnett, J., Mayer Jr., J.E., et al. (1997) Factors That Influence the Development of Atrial Flutter after the Fontan Operation. The Journal of Thoracic and Cardiovascular Surgery, 113, 80-86. https://doi.org/10.1016/S0022-5223(97)70402-1

[16] Olgin, J., Kalman, J., Fitzpatrick, A. and Lesh, M. (1995) Role of Right Atrial Endocardial Structures as Barriers to Conduction during Human Type I Atrial Flutter: Activation and Entrainment Mapping Guided by Intracardiac Echocardiography. Circulation, 92, 1839-1848. https://doi.org/10.1161/01.CIR.92.7.1839

[17] Hashimoto, K., Kurosawa, H., Tanaka, K., Yamagishi, M., Koyanagi, K., Ishii, S., et al. (1995) Total Cavopulmonary Connection without the Use of Prosthetic Material: Technical Considerations and Hemodynamic Consequences. The Journal of Tho- 
racic and Cardiovascular Surgery, 110, 625-632. https://doi.org/10.1016/S0022-5223(95)70093-5

[18] Sinha, P., Zurakowski, D., He, D., Yerebakan, C., Freedenberg, V., Moak, J. and Jonas, R. (2013) Intra/Extracardiac Fenestrated Modification Leads to Lower Incidence of Arrhythmias after Fontan Operation. Congenital Heart Disease, 145, 678-682.

[19] Gaies, M., Gurney, J., Yen, A., Napoli, M., Gajarski, R., Ohye, G., Charpie, J. and Hirsch, J. (2010) Vasoactive-Inotropici Score as a Predictor of Morbidity and Mortality in Infants after Cardiopulmonary Bypass. Pediatric Critical Care Medicine, 11, 234-238. https://doi.org/10.1097/PCC.0b013e3181b806fc

[20] Carins, T.A., Shi, W., Iyengar, A., et al. (2016) Long-Term Outcomes after First-Onset Arrhythmia in Fontan Physiology. The Journal of Thoracic and Cardiovascular Surgery, 152, 1355-1363. https://doi.org/10.1016/j.jtcvs.2016.07.073

[21] Ohuchi, H. (2017) Where Is the "Optimal" Fontan Hemodynamics? Korean Circulation Journal, 47, 842-857. https://doi.org/10.4070/kcj.2017.0105

[22] Ohuchi, H., Miyazaki, A., Negishi, J., Hayama, Y., Nakai, M., Nishimura, K., Ichikawa, H., Shiraishi, I. and Yamada, O. (2017) Hemodynamic Determinants of Mortality after Fontan Operation. American Heart Journal, 189, 9-18.

https://doi.org/10.1016/j.ahj.2017.03.020

[23] Hsia, T.Y., Migliavacca, F., Pittaccio, S., Radaelli, A., Dubini, G., Pennati, G. and de Leval, M. (2003) Computational Fluid Dynamic Study of Flow Optimization in Realistic Models of the Total Cavopulmonary Connections. Journal of Surgical Research, 116, 305-313. https://doi.org/10.1016/j.jss.2003.08.004 